\title{
Synthetic Studies on Soraphen A: Synthesis of the C1-C9 Hemiketal Segment
}

\author{
Se Hwan Park and Hỵo Won Lee

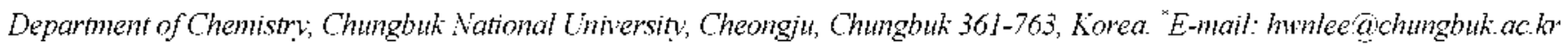 \\ Received April 30, 2008
}

Key Words : Soraphen A, Macrolide, Hemiketal, Aldol reaction. Crotylation

Soraphen A (1) isolated from myxobacterium Soranginm cellulostm exhibits potent antifungal activity against various pathogenic plant fungi. ${ }^{l}$ This polyketide has highly efficient inhibitory action on eukaryotic acetyl-coenzy'me A carboxylases (ACCs), and specifically their biotin carboxylase (BC) domains of ACCs. ${ }^{1}$ ACCs have cnucial roles in the metabolism of fatty acids and. therefore are targets for drug development against obesity. diabetes. and other diseases." This natural product contains an unsaturated 18 -membered lactone ring. an unsubstituted phenyl ring. and a hemiketal ring constituting its structural feature. ${ }^{3}$ There have been several synthetic studies reported in the literature. ${ }^{3}$ The first total sy'nthesis of soraphen A was reported by Giese in $1995^{\text {ja.l. }}$ We have reported our sy'nthetic studies. ${ }^{\text {jet }}$

We envisioned that it would be the synthesis of soraphen A via a sequence of the esterification and ring-closing metathesis (RCM) between $2^{3 e}$ and 3 segments (Scheme 1). Herein we disclose our efforts in the construction of the $\mathrm{Cl}$ C9 segment. General stereochemical control of carboxylic acid 3 was devised from the combined application of Evans aldol reaction ${ }^{4}$ and Roush asymmeric crotylation (Scheme 1).

As shown in Scheme 2, the synthesis of 3 began with Roush crotylation between $(E)$-crotylboronate 7 and aldehy'de 6. prepared from conmercially available $(5)-5,{ }^{6}$ to deliver homoally lic alcohol $8(82 \%, 97: 3 \mathrm{dr}){ }^{5}$ Removal of the TBS group in 8 under mild condition by TBAF followed by exposure to $p$-methoxybenzaldehyde dimethylacetal 9 and CSA yielded $p$-methoxyphenyl acetal 10 in good yield. Subsequent regioselective opening of acetal 10 using DIBAL provided the alcohol $11(90 \%)$ and the Swem oxidation upon 11 fumished the intennediate aldehyde Boron-mediated aldol reaction of the intermediate with oxazolidinone $12^{4}$ gave the desired aldol adduct $13(70 \%$ over two steps from 11.93:7 dr) with the requisite stereochemistry at $\mathrm{C} 4$ and $\mathrm{C} 5$ of soraphen A. Protection of the hydroxy group of $\mathbf{1 3}$ by treatment with TBSOTf and 2.6lutidine and reductive cleavage of the oxazolidinone moiety of the resulting TBS ether by $\mathrm{LiBH}_{4}$ yielded alcohol 14 in good yield. Similarly. the stereochemistry at $\mathrm{C} 2$ and $\mathrm{C} 3$ of soraphen A could be achieved using recurring Evans protocol. Consequently the intermediate aldehyde was obtained quantitatively from Parikh-Doering oxidation ${ }^{7}$ upon 14. Other oxidation conditions such as Swem oxidation and Dess-Martin oxidation provided in $43 \%$ and $48 \%$ yields. respectively. The aldehyde was submitted to the aldol reaction with the chiral oxazolidinone $15{ }^{+}$The resultant aldol adduct 4 ( $72 \%$ yield over two steps from 14 a single diastereomer) was transformed into hemiketal 16 by a twostep protocol, oxidation to the secondary alcohol with DessMatin periodinane and concomitant cyclization by oxidative cleavage the MPM group of 4 treating with DDQ ( $79 \%$ yield over two steps from 4 ).

At this stage. the structural configuration of hemiketal 16 was determined on the basis of 2D-NMR spectroscopic data (COSY. HMQC, and HMBC) along with relevant NOE studies (Scheme 2). As we expected. the six-membered ring shows a chair conformation with a methyl, a methoxy and a hydroxyl groups of the hemiketal ring occupying axial positions. which is concluded that the substituents possess the same orientation as in soraphen $\mathrm{A}^{\text {1a.31 }}$

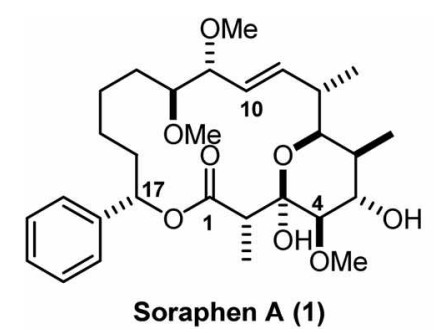

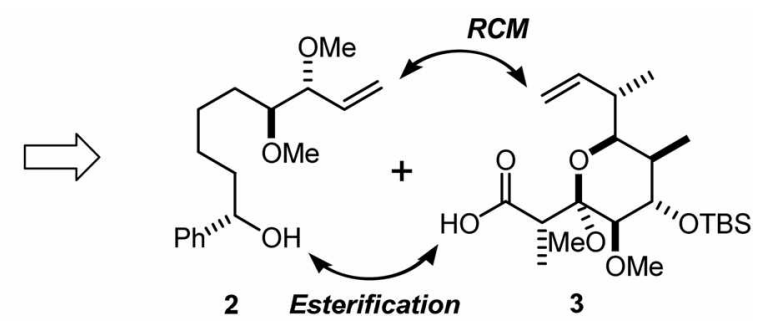<smiles>[B-]O[C@H]1[C@H](C)[C@@H]([C@@H](C)C=C)O[C@](C)(C(C)=O)[C@@H]1OC</smiles>

3

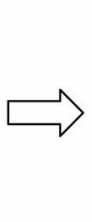

Scheme 1. Retrosynthetic Analysis.

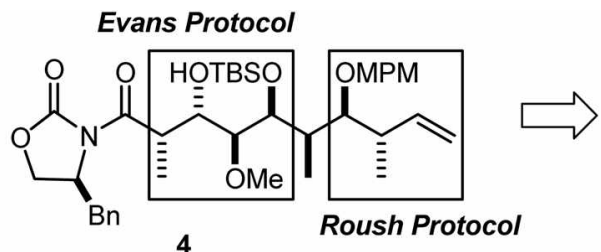

4<smiles>COC(=O)[C@H](C)CO</smiles> 


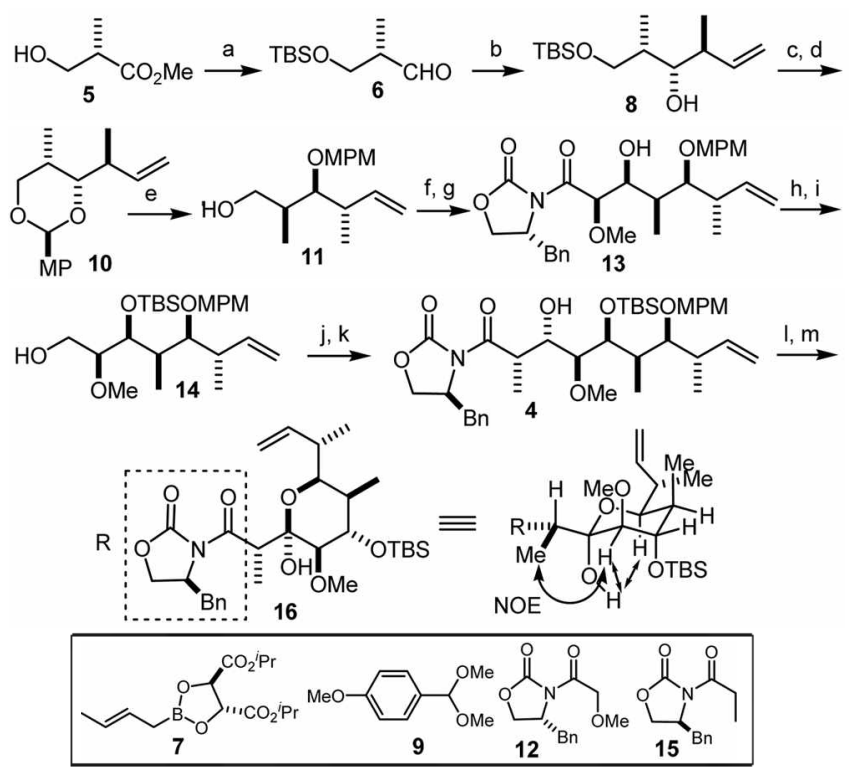

Scheme 2. Reaction Pathway to the Hemiketal 16. (a) Ref. 6. (b) 7 , 4A MS, MePl, $-78{ }^{\circ} \mathrm{C}, 3 \mathrm{~h}, 82 \%$. (c) TBAF, THF, rt, 1 h, $92 \%$. (d) 9, $\mathrm{CSA}, \mathrm{CH}_{2} \mathrm{Cl}_{2}, 0^{\circ} \mathrm{C}, 30 \mathrm{~min}, 88 \%$. (e) DIBAL-H, $\mathrm{CH}_{2} \mathrm{Cl}_{2}, 0^{\circ} \mathrm{C}, \mathrm{I}$ h, $90 \%$. (f) (CICO), DMSO, $上 t_{3} \mathrm{~N}, \mathrm{CH}_{2} \mathrm{Cl}_{2}, 1$ h. (g) $12, n-$ $\mathrm{Bu}_{2} \mathrm{BOTf}, \mathrm{Et}+\mathrm{N}, \mathrm{CH}_{2} \mathrm{Cl}_{2}, 3 \mathrm{~h}, 70 \%$ over two steps from 11. (h) TBSOTf, 2,6 -lutidine, $\mathrm{CH}_{2} \mathrm{Cl}_{2}, 2 \mathrm{~h}, 90 \%$. (i) $\mathrm{LiBH}_{4}, \mathrm{H}_{2} \mathrm{O}$, THF, 0 ${ }^{\circ} \mathrm{C}, 3$ h. $85 \%$. (i) $\mathrm{SO}_{3}$.py, $\mathrm{Et}_{3} \mathrm{~N}, \mathrm{DMSO} \cdot \mathrm{CH}_{2} \mathrm{Cl}_{2}(\mathrm{l}: 1), 0^{\circ} \mathrm{C}, 1 \mathrm{~h}$. (k) $15, n$-Bul- BOTf, $\mathrm{Et}_{3} \mathrm{~N}, \mathrm{CH}_{2} \mathrm{Cl}_{2}, 3 \mathrm{~h}, 72 \%$ over two steps from 14 . (l) DMP, $\mathrm{CH}_{2} \mathrm{Cl}_{2}$, it, $\mathrm{l}$ h, $92 \%$, (m) DDQ, $\mathrm{CH}_{2} \mathrm{Cl}_{2}, \mathrm{H}_{2} \mathrm{O}$, rt, $2 \mathrm{~h}, 86 \%$.

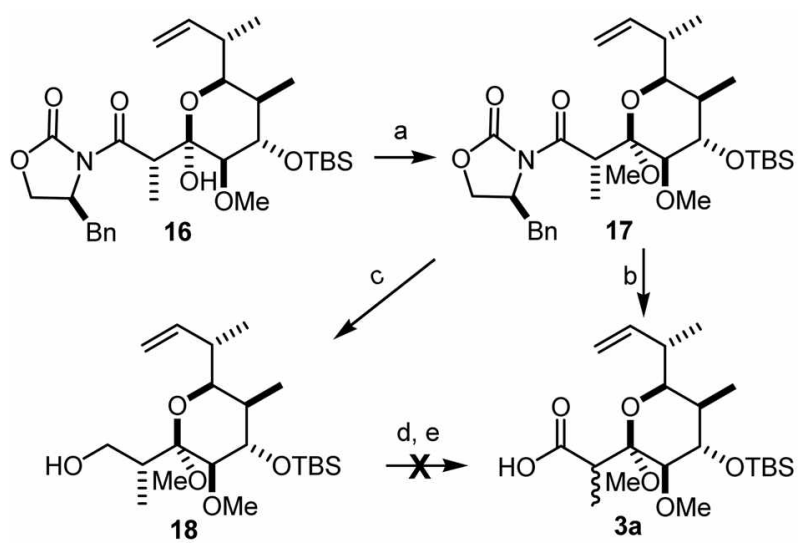

Scheme 3. Approach to the Desired Acid 3. (a) $\mathrm{HC}(\mathrm{OMe})_{3}: \mathrm{CH}_{2} \mathrm{Cl}_{2}$ (2:1), PPTS, rt, 48 h, 70\% (b) $\mathrm{LiOH} \cdot \mathrm{H}_{2} \mathrm{O}, \mathrm{H}_{2} \mathrm{O}_{2}, \mathrm{THF}, \mathrm{H}_{2} \mathrm{O}, 0$ " ${ }^{\prime \prime} \mathrm{C}, 3 \mathrm{~h}$, $40 \%$. (c) $\mathrm{NaBH}_{4}, \mathrm{THF}: \mathrm{H}_{2} \mathrm{O}(5: 1), 0^{\circ} \mathrm{C}, 3 \mathrm{~h}, 79 \%$ (d) $\mathrm{SO}_{3} \mathrm{py}, \mathrm{Et}_{3} \mathrm{~N}$, DMSO $\mathrm{CH}_{2} \mathrm{Cl}_{2}(1: 1), 0^{\circ} \mathrm{C}, 1$ h, (e) $\mathrm{NaClO}_{2}, \mathrm{NaH}_{2} \mathrm{PO}_{+}, 2$-methyl-2butene, THF: ${ }^{\mathrm{BuOH}}: \mathrm{H}_{2} \mathrm{O}(3: 3: 1), 0^{\circ} \mathrm{C}, 30 \mathrm{~min}$, decomposed.

With the desired hemiketal 16 in hand, the elaboration to the target acid 3 was required (Scheme 3). The anomeric hyddroxy group of 16 was converted to intermediate 17 by treatment with $\mathrm{HC}(\mathrm{OMe})_{s}$ and PPTS (70\%). Hydrolysis of 17 by the conventional treatment with $\mathrm{LiOOH}^{8}$ afforded an epinieric mixture $3 \mathbf{a}$ of the desired carboxylic acid (40\%). The epimerization at this step was unavoidable under our condition. However. other hydrolysis conditions employing aqueous $\mathrm{KOH}$ and $\mathrm{NaOH}$ ended up to give us poor conversion. As an alternative measure we performed the reductive cleavage of the chiral auxiliary of intermediate 17 by $\mathrm{NaBH}_{4}$ to obtain primary alcohol $18(79 \%)$. Thus we expected the oxidation of 18 by Parikh-Doering oxidation would give the intermediate aldehyde. which could be readily oxidized with $\mathrm{NaClO}_{2}$ to carboxylic acid 3. However. we could not advance in the desired direction. The aldelyde was found to immediately decompose when we carried out oxidation with $\mathrm{NaClO}_{2}$.

In summary. the Cl-C9 segment of soraphen A with C.2 epimer was prepared wia 17 -step sequence in $4.6 \%$ overall yield. Successfully we was introduced the seven stereogenic centers using Evans aldol reaction and Roush asymmeric crotylation and the desired hemiketal ring.

Acknowledgments. This work was supported by the research grant of the Chungbuk National University in 2006.

\section{References}

1. (a) Bedorf. N.: Schomburg. D.: Gerth. K:: Reichenbach. H. Hofle, G. Liebigs Ann. Chem. 1993. 1017-1021. (b) Gerth. K: Reichenbach, H.: Bedorf. N.: Irschik, H.: Hotle, G. $J$. Antibiotics 1994. 17.23-31. (c) GBF $\mathrm{mbH}$ and Ciba-Geigy $A G$ EP $0282455 \mathrm{~A} 3$.

2. Shen. Y: Volrath. S. L:: Weatherly. S. C.: Elich. T. D.: Tong. L. Molecula Cell 2004. $16(6) .881-891$

3. (a) Abel. S.: Faber. D.: Huter. O.: Giese. B. Angew. Chen., Int. Ed. Engl. 1994. 33. 2466-2468. (b) Abel. S.: Faber. D.: Huter. O. Giese. B. Simthesis 1999, 188-197. (c) Vincent, G. Mansfield, D. J. Vors, J.-P. Ciufolini, M. A. Ong. Lett. 2006. 11, 2791-2794. (d) Diaz-Oltra, S.: Murga. J.; Falomir, E: Carda. M.: Peris. G: Marco. T. A. J. Org. Chem 2005. 70. 8130-8139. (e) Lee. H. W. Kim. Y. J. Bul. Kowan Chem. Soc. 1996. 17. 1107-1108. (f) Lee. H. W.: Lee. I.-C.: Kim. Y.-S.: Park. S.-U. Bull. Konean Chem. Soc. 2002. 23, 1197-1198. (g) Park. S. H.: Lee. H. W.: Park. S.-U. Bull. Korean Chem. Soc, 2004. 25, 1613-1614. (h) Gurjar, M. K. Mainkar. A. S.: Srinivas, P. Tetrohedron Lett. 1995. 36, $5967-$ 5968. (i) Cao. Y.: Eweas. A. F.: Donaldson. W. A. Tetrahedron Lett. 2002. $13.7831-7834$. (j) Loubinoux. B.: Sinnes. T.-L:: O'Sullivan. A. C.: Winkler. I. Heh: Chint. Acta 1995. 78. 122-128. (k) Loubinoux, B.; Sinnes, J.-L; O'Sullivan, A. C.: Winkler, T. Tetahedron 1995, 51. 3549-3558. (1) Loubinoux. B. Sinnes, J.-L.: O'Sullivan, A. C.; Winkler. T. J. Org Chem 1995. 60,953-959.

4. (a) Gage. T.: Evans. D. A. Org. Smm 1990. 68. 83-91. (b) Evans. D. A.: Bartoli. J.: Shih. T. L. J. An. Chem. Soc. 1981. 103. $2127-$ 2129. (b) Danda. H.: Hansen. M. M.: Heathcock. C. H. J. Org. Chem. 1990. 55. 173-181. (c) Evans. D. A.: Black. W. C. J. Am. Chem. Soc. 1993, 115, 4197-4513. (d) Romo. D.: Johnson, D. D: Plamondon, L. Miwa, T.: Schreiber. S. L. J. Org. Chem. 1992, 57. 5060)-5063. (e) Hwang. T. M.: Yeom. S. H: Jun1g. K. Y. Bwll. Korean Chem. Soc. 2007. 28.821-826

5. (a) Roush. W. R: Ando. K: Power. D. B.: Palkowitz. A. D.: Halterman, R. L. d. Am. Chm. Soc. 1990, 112. 6339-6348. (b) Roush. W. R.: Ando. K.: Power. D. B.: Palkowitz, A. D. J. Am. Chem. Soc. 1990, 112, 6348-6359. (c) Roush, W. R.: Hoong, L. K.: Palmer. M. A.: Straub. J. A.: Palkowitz. A. D. J. Org. Chent. 1990. 55. 4117-4126. (d) Yang. H.-H.: Kim. E.-S.: Yoon. Y. J.: Kang. H.-Y. Bull. Korean Chem. Soc. 2006. 27. 473-474.

6. (a) Jones, T. K.: Reamer. R. A : Desmond, R.; Mills. S. G. J. Am. Chem. Soc. 1990, 112. 2998-3017. (b) Paik. S. Bull. Koman Chen. Soc. $1999,20.217-219$

7. (a) Parikih. J. P.: Doering. W. E. J. Am. Chent. Soc. 1967.89.55055507. (b) Panek. J. S.: Masse. C. E. J. Org. Chent 1997. 62.82908291 .

8. (a) Gage. J. R. Evans. D. A. Ong. Synth. 1989. 68, 83-91. (b) Evans. D. A.: Britton. T. C.: Ellman. J. A. Tetrahedron Latt. 1987. 28. $6141-61+4$. 